\title{
Commentary
}

\section{Community paramedicine in British Columbia: A virtual response to COVID-19}

Michelle Brittain is Project Manager of Strategic \& Process Initiatives ${ }^{1}$; Christopher Michel is a community paramedic mentor ${ }^{1}$ and sessional academic'; Leon Baranowski MSc, MEd, FHEA, is Paramedic Practice Leader ${ }^{1}$; Richard Armour BParamedPrac is an Advanced Care Paramedic ${ }^{1}$ and sessional academic ${ }^{2,3}$; Jennie Helmer BCom, MEd is Research Lead ${ }^{1}$, sessional academic ${ }^{2}$ and PhD student'; Amy Poll is Interim Director of Strategic Program Development ${ }^{1}$

\section{Affiliations:}

${ }^{1}$ British Columbia Emergency Health Services, Vancouver, Canada

2Justice Institute of British Columbia, Vancouver, Canada

${ }^{3}$ Charles Sturt University, Bathurst, New South Wales

${ }^{4}$ University of British Columbia, Vancouver, Canada

https://doi.org/10.33151/ajp.17.813

\section{Abstract}

The COVID-19 pandemic has forced innovative approaches to patient care delivery. The British Columbia Emergency Health Service has worked collaboratively with health authorities throughout the province since 2015 to improve the delivery of healthcare in rural and remote communities through the community paramedicine program. In response to the COVID-19 pandemic to minimise the risk to providers and patients, as well as conserve personal protective equipment, home visits and community engagement opportunities were suspended. However, the COVID-19 pandemic saw a large increase in the number of patients referred to the service and so alternate approaches to patient care delivery were urgently required. This commentary outlines the integration of home health monitoring technology into the community paramedicine program within British Columbia as well as the integration of virtual, telehealth consultations in response to the COVID-19 pandemic.

\section{Keywords:}

paramedic; community paramedicine; rural health; community paramedic; telehealth; technology

Corresponding Author: Michelle Brittain, Michelle.Brittain@bcehs.ca 


\section{Introduction}

The COVID-19 pandemic and the associated physical distancing measures to minimise the spread of the infection have forced innovative approaches to patient care delivery. Virtual health was first explored as an approach to healthcare delivery in Canada during the 1970s, with Dr House of Memorial University, Newfoundland providing telephone consultations to remote sites within the province of Newfoundland (1). Although virtual healthcare delivery has since seen a progressive adoption, during the COVID-19 pandemic there has been a rapid expansion across areas of healthcare that had previously seen little uptake of technology for the delivery of services (2). Since the declaration of the pandemic, British Columbia has rapidly expanded its virtual health footprint throughout the province led by the Office of Virtual Health under the Provincial Health Service Authority (PHSA). The British Columbia Emergency Health Service (BCEHS) falls under the jurisdiction of PHSA and was required to rapidly innovate means for delivering the already established community paramedicine program (CPP) across the province, which are outlined in this commentary.

\section{Community paramedicine in British Columbia}

BCEHS is the primary provider of emergency out-of-hospital health services across the province of BC, covering 950,000 square kilometres and a population of 5 million people (3). The growing and ageing population within the province has increased the demand on paramedics, and also on primary and emergency healthcare professionals. In an effort to reduce pressures on the entire healthcare system, specifically in rural and remote communities with challenges to accessing primary care, a CPP was developed in 2015. This program aimed to shift the paradigm of out-of-hospital emergency care from rapid triage, clinical care and transfer to hospital, towards a holistic model of paramedic-led healthcare delivery. The CPP aims to work collaboratively with the broader healthcare system in the prevention of illness and with a focus on health promotion in rural and remote communities. Community paramedicine was introduced to an initial 99 rural and remote communities within $B C$ with limited access to healthcare, as identified by the Ministry of Health in conjunction with regional stakeholders. The program has continued to grow, and in 2019 there were 19,306 scheduled community paramedic visits delivered, of which 45 were delivered on a trial basis via telehealth, as well as 8341 community outreach and community health promotion services.

The majority of community paramedicine services in $B C$ are delivered by Primary Care Paramedics with additional practice endorsements, with an additional five Advanced Care Paramedic community paramedics. Paramedics complete an internal 9-week orientation to practise as a community paramedic, in addition to education delivered in partnership with the Justice Institute of British Columbia related to public and primary health systems, principles of chronic disease management, and the community paramedic's role in health promotion, prevention, screening and monitoring within the community.

As the CPP has grown it has expanded to meet the individual needs of the communities it serves. Currently, the program provides care and support for seniors living with chronic diseases such as heart disease, diabetes, chronic obstructive pulmonary disease and hypertension, as well as those at risk of falls, at no cost to the patients. These services are focussed around supporting patients in managing their health in a way that allows them to continue to live safely in their home. Community paramedics deliver patient education around chronic conditions; ensure the patient has a comprehensive fall risk and safety assessment; support medication management; ensure the patient is sufficiently connected with their primary care physician or nurse practitioner, assisting the patient in navigating the health system when additional care is required. These services were bolstered in 2017 with the implementation of a remote patient monitoring service, Home Health Monitoring. This technology allowed community paramedics to monitor key vital signs, symptoms and health indicators in patients from rural and remote communities and improve patient and system outcomes by enabling more tailored management of chronic conditions within the home (4).

\section{A virtual response to COVID-19}

In order to minimise the risk to providers and patients and to conserve personal protective equipment, regular face-to-face community paramedic home visits and community engagement opportunities were suspended in March 2020 (5). Paradoxically, this time saw a large increase in requests for community paramedicine services from allied health professionals with an additional 424 patient enrolments bringing the total number of patients enrolled in the CPP to 1341. From these 1341 patients there were approximately 700 weekly assessments performed, and so a clear need for innovation in the delivery of services to meet this demand was identified. Initially, community paramedics shifted to delivering services via telephone consultations with patients. However, this was subsequently expanded to include the use of video conferencing for both patient assessment as well as community education events (6). With the introduction of this technology, community paramedics have been able to increase the frequency of engagement with identified at-risk patients using both Zoom for Healthcare and FaceTime applications to collaborate with the patient and their primary care physician and allied health team members (7). Since the beginning of the COVID-19 telehealth initiative for community paramedicine services on 23 March there have been 3897 consultations successfully completed using telehealth solutions. These changes were supported by both local policy development as well as provincial legislation $(6,7)$ and were solidified by the development of virtual health clinical practice guidelines (8). These guidelines provide community paramedics with a framework for a telehealth consultation as proposed by Greenhalgh et al $(8,9)$, providing a model for remote clinical assessment that highlights clinical presentations 
requiring emergency responses $(9,10)$. Additionally, in an effort to curb community transmission of COVID-19, community paramedics now screen patients for potential symptoms of COVID-19 as well as monitor for signs of COVID-19 through the Home Health Monitoring initiative.

However, despite the apparent success of these changes, in practice a number of issues previously described in the literature were encountered by BCEHS community paramedics $(9,11,12)$. This has included limitations in accessing telehealth related to the availability and accessibility of broadband internet access in rural and remote regions in BC. Reduced technological ability and physical ailments, such as poor hearing or eyesight, have also been noted to influence the successful delivery of BCEHS community paramedicine services by telehealth, with paramedics required to use clinical judgement to determine the safest and most appropriate method of communication, assessment and care delivery for each patient $(9,12)$. As the pandemic continues, necessitating the ongoing delivery of BCEHS CPP services by telehealth, research will be conducted by BCEHS community paramedics to better understand the unique needs of patient populations receiving telehealth and how these services can be tailored to meet the needs of these patient populations.

\section{The future of telehealth in community paramedicine} COVID-19 has fundamentally changed the way community paramedicine services are delivered in $\mathrm{BC}$, possibly even beyond the conclusion of the pandemic. The virtual community paramedic response has supported BCEHS and provincial strategic goals in response to COVID-19, as well as improving paramedic safety and increasing system capacity. It is too early to tell how exactly this may integrate into future care however, the flexibility virtual care brings to service delivery allows for patient assessment from across the province while improving patient connection with the broader healthcare system. This may also be applicable in ensuring appropriate patient re-engagement for safeguarding as paramedic services increase community referral from 9-1-1 emergency calls. Systems utilising telehealth for the delivery of community paramedicine services should consider engaging in research and collaborative communities of practice around the use of telehealth to evaluate the potential future of telehealth in global community paramedicine service delivery.

\section{Competing interests}

The authors declare no competing interests. Each author of this paper has completed the ICMJE conflict of interest statement.

\section{References}

1. House A, Roberts J. Telemedicine in Canada. CMAJ 1977; 117:386-8.

2. Khairat S, Meng C, Xu Y, Edson B, Gianforcaro R. Interpreting COVID-19 and virtual care trends: cohort study. JMIR Public Health Surveill 2020;15:18811. doi: 10.2196/18811

3. British Columbia Central Statistics Agency. Quarterly population highlights. Victoria, British Columbia; January 2020. Report No: 19-04. Available at: www2.gov.bc.cal assets/gov/data/statistics/people-population-community/ population/population_highlights_2019q4.pdf

4. Island Health Authority. Evaluation of the Home Health Monitoring Expansion Project. Victoria, British Columbia; March 2018. Available at: www.infoway-inforoute.ca/en/ component/edocman/3482-evaluation-of-the-home-healthmonitoring-expansion-project/view-document? ltemid $=0$

5. Chauhan V, Galwankar S, Arquilla B, et al. Novel coronavirus (COVID-19): leveraging telemedicine to optimize care while minimizing exposures and viral transmission. J Emerg Trauma Shock 2020;13:20-4. doi: 10.4103/JETS.JETS_32_20

6. Kang A. Freedom of Information and Protection of Privacy Act, Ministerial Order. Victoria, British Columbia; March 2020. Report No: M085. Available at: www.bclaws.ca/civix/ document/id/mo/mo/2020_m085

7. Provincial Health Services Authority. Virtual Health COVID-19 Accessible Solution Toolkit. Vancouver, British Columbia; March 2020. Available at: www. phsa.ca/Documents/Trauma-Services/PHSA_ VirtualHealthToolkit_031920.pdf

8. British Columbia Emergency Health Services. Clinical Practice Guidelines Handbook. Vancouver, British Columbia; March 2020. Available at: http://handbook.bcehs. ca

9. Greenhalgh T, Koh G, Car J. COVID-19: a remote assessment in primary care. BMJ 2020;368:m1182. doi: 10.1136/bmj.m1182

10.Schroeder K, Chan W, Fahey T. Recognising red flags in general practice. InnovAIT 2011;4:171-6. doi: 10.1093/ innovait/inq143

11. Poole L, Brown H, Brooksbank K, Amar C, Verma J. Bridging the gap between patients and providers through telehealth in rural and remote regions [webinar series]. Canadian Foundation for Healthcare Improvement; June 2015. Available at: www.cfhi-fcass.ca/sf-docs/ default-source/on-call/2015-06-10-presentation. pdf?sfvrsn=870bde44_2

12. Martich $\mathrm{D}$. Telehealth nursing: tools and strategies for optimal patient care. New York, New York: Springer Publishing Company; 2017. 